\title{
THE EXISTENCE OF LEFT AVERAGING FUNCTIONS THAT ARE NOT RIGHT AVERAGING
}

\author{
TIANXUAN MIAO
}

(Communicated by J. Marshall Ash)

\begin{abstract}
Let $G$ be a locally compact group. We show that $G$ is amenable as a discrete group if and only if $\sum_{i=1}^{n} \lambda_{i x_{i}} f \in \mathscr{A}_{0}$ for any $f_{0} \in \mathscr{A}_{0}, x_{i} \in G$, and $\lambda_{i}>0 \quad(i=1,2, \ldots, n)$ with $\sum_{i=1}^{n} \lambda_{i}=1$, where $\mathscr{A}_{0}$ is the set of functions that left average to 0 . We also confirm a conjecture of Rosenblatt and Yang that there is a left averaging function that is not right averaging if $G$ is not amenable.
\end{abstract}

Let $G$ be a locally compact group with a fixed left Haar measure $\lambda$. Let $L^{p}(G)$ be the associated real Lebesgue spaces $(1 \leq p \leq \infty)$. For each $f \in$ $L^{\infty}(G)$ and $x \in G,{ }_{x} f \in L^{\infty}(G)$ is defined by ${ }_{x} f(y)=f(x y), y \in G$. Similarly, we can define $f_{x} \in L^{\infty}(G)$ by $f_{x}(y)=f(y x), y \in G$. For $f \in$ $L^{\infty}(G)$ and a constant $c$, we say that $f$ left averages to $c$ if $c \in\|\|_{\infty}$-closed convex hull of $\left\{{ }_{x} f: x \in G\right\}$. Let $\mathscr{A}$ denote the set of all functions that left average to some constant, and let $\mathscr{A}_{0}$ denote the set of functions that left average to 0 . Similarly, we say that $f$ right averages to $c$ if $c \in\|\|_{\infty}$-closed convex hull of $\left\{f_{x}: x \in G\right\}$, and we denote the set of all the right averaging functions by $\mathscr{A}_{R}$. A positive linear functional of norm 1 on $L^{\infty}(G)$ is called a mean. A mean $m$ is said to be left invariant if $m\left({ }_{x} f\right)=m(f)$ for any $x \in G$ and $f \in L^{\infty}(G)$. We denote the set of all left invariant means by $\operatorname{LIM}(G) . G$ is said to be amenable if $\operatorname{LIM}(G) \neq \varnothing$. It is well known that $G$ is amenable if $G$ is amenable as a discrete group, but the converse may fail (see [1]).

It is natural to ask if every left averaging function is right averaging. This problem was first studied by Rosenblatt and Yang [3]. They showed that when $G$ is amenable as a discrete group, $\mathscr{A}=\mathscr{A}_{R}$ if and only if $\operatorname{LIM}(G)=\operatorname{RIM}(G)$, where $\operatorname{RIM}(G)$ is the set of all right invariant means i.e. the mean $m$ with $m\left(f_{x}\right)=m(f)$ for any $x \in G$ and $f \in L^{\infty}(G)$. They conjectured that when $G$ is not amenable, there is a left averaging function that is not right averaging.

In this note we prove that $G$ is amenable as a discrete group if and only if $\sum_{i=1}^{n} \lambda_{i} x_{i} f \in \mathscr{A}_{0}$ for any $f \in \mathscr{A}, x_{i} \in G$, and $\lambda_{i}>0$ with $\sum_{i=1}^{n} \lambda_{i}=1$.

Received by the editors September 17, 1990 and, in revised form, November 8, 1990.

1980 Mathematics Subject Classification (1985 Revision). Primary 43A07.

Key words and phrases. Locally compact group, amenable group, invariant means, the left averaging functions.

This research was supported in part by the Killam Memorial Scholarship at the University of Alberta. 
This improves the main result of Miao [2]. Then we apply this theorem to confirm the conjecture of Rosenblatt and Yang. To prove our result, we need the following lemmas.

Lemma A. (a) For $f \in L^{\infty}(G)$, let $\check{f} \in L^{\infty}(G)$ be defined by $\check{f}(x)=f\left(x^{-1}\right)$ $(x \in G)$. Then $f \in \mathscr{A}$ if and only if $\check{f} \in \mathscr{A}_{R}$.

(b) $\mathscr{A} \subseteq A_{R}$ if and only if $\mathscr{A}=\mathscr{A}_{R}$.

Proof. (a) For any $x \in G$ and $t \in G$,

$$
\left({ }_{x} \check{f}\right)(t)=f\left(x t^{-1}\right)=\check{f}\left(t x^{-1}\right)=\check{f}_{x^{-1}}(t) .
$$

Hence $\left({ }_{x} \check{f}\right)=\check{f}_{x^{-1}}$. It is easy to see that the following are equivalent: (i) $f \in \mathscr{A}$; (ii) there is a constant $c$ such that $c \in \overline{\text { convex }}^{\|} \|_{\infty}\left\{{ }_{x} f: x \in G\right\}$; (iii) $c \in \overline{\text { convex }}^{\|}\left\|_{\infty}\left\{\left({ }_{x} \check{f}\right): x \in G\right\}=\overline{\text { convex }}\right\|^{\|} \|_{\infty}\left\{\check{f}_{x^{-1}}: x \in G\right\}$; and (iv) $\check{f} \in \mathscr{A}_{R}$.

(b) Let $\mathscr{A} \subseteq \mathscr{A}_{R}$. If $f \in \mathscr{A}_{R}$ then $\check{f} \in \mathscr{A}$ by (a) since $(\check{f})=f$. Hence $\check{f} \in \mathscr{A} \subseteq \mathscr{A}_{R}$ and $f \in \mathscr{A}$ by (a) again. Therefore $\mathscr{A}=\mathscr{A}_{R}$.

Lemma B. $\mathscr{A}_{0}$ is a subspace if and only if for any $f \in \mathscr{A}_{0}, \sum_{i=1}^{n} \lambda_{i x_{i}} f \in \mathscr{A}_{0}$ for any $x_{i} \in G, \lambda_{i}>0(i=1,2, \ldots, n)$ with $\sum_{i=1}^{n} \lambda_{i}=1$.

Proof. Suppose that $\mathscr{A}_{0}$ is a subspace. If $f \in \mathscr{A}_{0}$, then ${ }_{x} f \in \mathscr{A}_{0}$ for any $x \in G$. Let $x_{i} \in G$ and $\lambda_{i}>0(i=1,2, \ldots, n)$, then $\lambda_{i x_{i}} f \in \mathscr{A}_{0}(i=1,2, \ldots, n)$. So $\sum_{i=1}^{n} \lambda_{i x_{i}} f \in \mathscr{A}_{0}$ because $\mathscr{A}_{0}$ is a subspace.

Conversely, let $\sum_{i=1}^{n} \lambda_{i x_{i}} f \in \mathscr{A}_{0}$ for any $f \in \mathscr{A}_{0}, x_{i} \in G$, and $\lambda_{i}>0 \quad(i=$ $1,2, \ldots, n)$ with $\sum_{i=1}^{n} \lambda_{i}=1$. It suffices to show that $\mathscr{A}_{0} \supseteq$ $\operatorname{span}\left\{{ }_{x} f-f: f \in L^{\infty}(G), x \in G\right\}$ by Lemma 2.1 in [2]. For $f_{1} \in L^{\infty}(G)$ and $x_{1} \in G, x_{1} f-f \in \mathscr{A}_{0}$. Let $F=\sum_{i=1}^{n-1} a_{i}\left(x_{i} f_{i}-f_{i}\right) \in \mathscr{A}_{0}$ for any constants $a_{i}, x_{i} \in G$ and $f_{i} \in L^{\infty}(G) \quad(i=1,2, \ldots, n-1)$. If $x_{n} \in G$ and $f_{n} \in L^{\infty}(G)$, then $\sum_{i=1}^{n} a_{i}\left(x_{i} f_{i}-f_{i}\right) \in \mathscr{A}_{0}$ where $a_{n}$ is a nonzero constant. Indeed, let $\varepsilon>0$. Since $x_{n} f_{n}-f_{n} \in \mathscr{A}_{0}$, there are $\lambda_{k}>0, y_{k} \in G(k=1,2, \ldots, N)$ such that $\sum_{k=1}^{N} \lambda_{k}=1$ and

$$
\left\|\sum_{k=1}^{N} \lambda_{k y_{k}}\left(x_{n} f_{n}-f_{n}\right)\right\|_{\infty}<\frac{\varepsilon}{2\left|a_{n}\right|} .
$$

Since $\sum_{k=1}^{N} \lambda_{k y_{k}} F \in \mathscr{A}_{0}$, there are $w_{l}>0$ and $z_{l} \in G(l=1,2, \ldots, L)$ such that $\sum_{l=1}^{L} w_{l}=1$ and

$$
\left\|\sum_{l=1}^{L} w_{l z_{l}}\left(\sum_{k=1}^{N} \lambda_{k y_{k}} F\right)\right\|_{\infty}<\frac{\varepsilon}{2} .
$$

Hence

$$
\begin{aligned}
\| \sum_{l=1}^{L} & w_{l z_{l}}\left[\sum_{k=1}^{N} \lambda_{k y_{k}}\left(\sum_{i=1}^{n} a_{i}\left(x_{i} f_{i}-f_{i}\right)\right)\right] \|_{\infty} \\
\leq & \left\|\sum_{l=1}^{L} w_{l z_{l}}\left(\sum_{k=1}^{N} \lambda_{k y_{k}} F\right)\right\|_{\infty}+\left|a_{n}\right| \sum_{l=1}^{L} w_{l}\left\|\sum_{k=1}^{N} \lambda_{k y_{k}}\left(x_{n} f_{n}-f_{n}\right)\right\|_{\infty} \\
& <\varepsilon / 2+\varepsilon / 2=\varepsilon .
\end{aligned}
$$

By induction, $\mathscr{A} \supseteq \operatorname{span}\left\{{ }_{x} f-f: x \in G, f \in L^{\infty}(G)\right\}$. 
Theorem C. For a locally compact group $G, G$ is amenable as a discrete group if and only if $\sum_{i=1}^{n} \lambda_{i x_{i}} f \in \mathscr{A}_{0}$ for any $f \in \mathscr{A}_{0}, \lambda_{i}>0$, and $x_{i} \in G$ with $\sum_{i=1}^{n} \lambda_{i}=1$

Proof. This is a direct consequence of Theorem 2.3 of [2] and Lemma B.

The following theorem confirms the conjecture of Rosenblatt and Yang [3]:

Theorem D. If $\mathscr{A} \subseteq \mathscr{A}_{R}$, then $G$ is amenable as a discrete group.

Proof. By Theorem C, it suffices to show that $\sum_{i=1}^{n} \lambda_{i x_{i}} f \in \mathscr{A}_{0}$ for any $f \in \mathscr{A}_{0}$, $x_{i} \in G$, and $\lambda_{i}>0(i=1,2, \ldots, n)$ with $\sum_{i=1}^{n} \lambda_{i}=1$. Since $\mathscr{A}_{0} \subseteq \mathscr{A} \subseteq \mathscr{A}_{R}$, $f \in \mathscr{A}_{R}$ and it right averages to 0 by Corollary 1.4 of [3]. Hence for any $\varepsilon>0$, there are $\beta_{k}>0, y_{k} \in G(k=1,2, \ldots, N)$ such that $\sum_{k=1}^{N} \beta_{k}=1$ and $\left\|\sum_{k=1}^{N} \beta_{k} f_{y_{k}}\right\|_{\infty}<\varepsilon$. Not that for each $i=1,2, \ldots, n$,

$$
\left\|x_{i}\left(\sum_{k=1}^{N} \beta_{k} f_{y_{k}}\right)\right\|_{\infty}=\left\|\sum_{k=1}^{N} \beta_{k}\left(x_{i} f\right)_{y_{k}}\right\|_{\infty}<\varepsilon .
$$

Hence

$$
\begin{aligned}
\left\|\sum_{k=1}^{N} \beta_{k}\left(\sum_{i=1}^{n} \lambda_{i x_{i}} f\right)_{y_{k}}\right\|_{\infty} & =\left\|\sum_{i=1}^{n} \lambda_{i}\left(\sum_{k=1}^{N} \beta_{k}\left(x_{i} f\right)_{y_{k}}\right)\right\|_{\infty} \\
& \leq \sum_{i=1}^{n} \lambda_{i}\left\|\sum_{k=1}^{N} \beta_{k}\left(x_{i} f\right)_{y_{k}}\right\|_{\infty}<\varepsilon,
\end{aligned}
$$

that is $\sum_{i=1}^{n} \lambda_{i x_{i}} f$ right averages to 0 . By Lemma $\mathrm{A}, \sum_{i=1}^{n} \lambda_{i x_{i}} f \in \mathscr{A}$. Hence $\sum_{i=1}^{n} \lambda_{i x_{i}} f$ left averages to 0 by Corollary 1.4 of [3] again. Therefore, $\sum_{i=1}^{n} \lambda_{i x_{i}} f \in \mathscr{A}_{0}$.

\section{REFERENCES}

1. F. P. Greenleaf, Invariant means on topological groups, Van Nostrand, New York, 1969.

2. T. Miao, Amenability of locally compact groups and subspaces of $L^{\infty}(G)$, Proc. Amer. Math Soc. (to appear).

3. J. M. Rosenblatt and Z. Yang, Functions with a unique invariant mean value, Illinois J. Math. 34 (1990), 744-764.

Department of Mathematics, University of Alberta, Edmonton, Alberta T6G 2G1 CANADA 